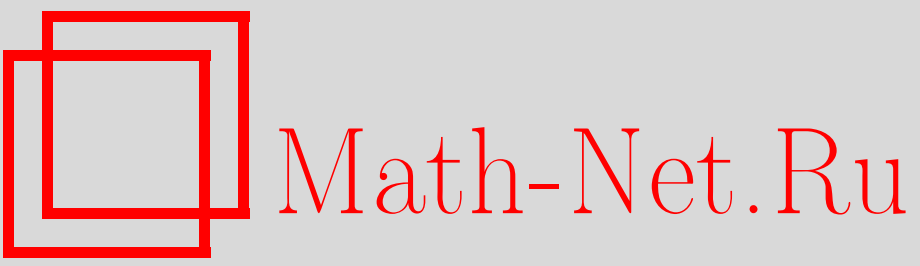

Б. Г. Фрейнкман, Самосогласованный расчет основного состояния водородоподобного атома углерода в решетке графена, Матем. моделирование, 2020, том 32, номер 8, 21-30

DOI: https://doi.org/10.20948/mm-2020-08-02

Использование Общероссийского математического портала Math-Net.Ru подразумевает, что вы прочитали и согласны с пользовательским соглашением

http: //www . mathnet.ru/rus/agreement

Параметры загрузки:

IP : 34.227 .88 .159

26 апреля 2023 г., 16:50:35 


\title{
САМОСОГЛАСОВАННЫЙ РАСЧЕТ ОСНОВНОГО СОСТОЯНИЯ ВОДОРОДОПОДОБНОГО АТОМА УГЛЕРОДА \\ В РЕШЕТКЕ ГРАФЕНА
}

\author{
(C) 2020 2. Б.Г. Фрейнкман \\ Институт прикладной математики им. М.В. Келдыша РАН \\ bgfreink@gmail.com
}

Работа выполнена при поддержке РФФИ, проект 20-07-00790-а.

DOI: $10.20948 / \mathrm{mm}-2020-08-02$

С открытия графена стартовала эра освоения принципиально новых материалов. Их уникальные свойства уже позволяют создавать множество полезных изделий в электронике, биомедицине и других высокотехнологичных отраслях промышленности. Однако изучение графена и его производных продолжается. До сих пор не до конца понятен механизм образования решетки графена и параметры состояния отдельных ее атомов. Причиной тому служит тот факт, что собственно графен невозможно получить, не положив атомы углерода на поверхность с определенными свойствами. Но в этом случае свойства графена существенно затеняются свойствами самой поверхности. Настоящая работа посвящена созданию модели графена в виде решетки водородоподобных атомов углерода. При этом используется модификация подхода Брандта-Китагавы с экранированными ионами, предложенная нами ранее. В приближении холодной решетки эта модель предполагает, что три валентных атома, ориентированных по линиям связи, принадлежат экранирующей оболочке иона. И только один валентный электрон определяет основное состояние атома решетки и неоднородное угловое распределение его поля.

Ключевые слова: решетка графена, водородоподобный атом, основное состояние, самосогласованное решение уравнения Шредингера, неоднородное центральное поле.

\section{SELF-CONSISTENT CALCULATION OF THE GROUND STATE OF A HYDROGEN-LIKE CARBON ATOM IN A GRAPHENE LATTICE}

\section{B.G. Freinkman}

Keldysh Institute of Applied Mathematics of RAS

With the discovery of graphene, the era of the development of fundamentally new materials began. Their unique properties already allow you to create many useful products in electronics, biomedicine and other high-tech industries. However, the study of graphene and its derivatives continues. The mechanism of the formation of the graphene lattice and the state parameters of its individual atoms are still not fully understood. The reason for this is the fact that graphene itself cannot be obtained without 
laying carbon atoms on a surface with certain properties. But in this case, the properties of graphene are substantially obscured by the properties of the surface itself. This work is devoted to the creation of a graphene model in the form of a lattice of hydrogen-like carbon atoms. In this case, we use a modification of the Brandt-Kitagawa approach with shielded ions that we proposed earlier. In the cold lattice approximation, this model assumes that the three valence atoms oriented along the bond lines belong to the ion shield. And only one valence electron determines the ground state of the lattice atom and the inhomogeneous angular distribution of its field.

Key words: graphene lattice, hydrogen-like atom, ground state, self-consistent solution of the Schrodinger equation, inhomogeneous central field.

\section{1. Введение}

Настоящая работа посвящена созданию модели графена в виде решетки водородоподобных атомов углерода. При этом используется модификация подхода Брандта-Китагавы [1] с экранированными ионами, предложенная нами ранее [2]. В приближении холодной решетки эта модель предполагает, что три валентных атома, ориентированных по линиям связи, принадлежат экранирующей оболочке иона. И только один валентный электрон определяет основное состояние атома решетки и неоднородное угловое распределение его поля.

В данной работе исследуется применимость подхода экранированного иона в модели Брандта-Китагавы к атому в решетке. Ранее, в численных экспериментах [3-5], было установлено, что поле иона неоднородно по радиусу, и это сильно влияет на точность определения энергии квантового состояния электрона. Эта неоднородность определяется параметром экранирования иона $\lambda_{0}$, который в работе [2] определяется для атомов и ионов в окружении равновесного электронного газа. В данной работе предложен алгоритм определения параметра экранирования, учитывающего энергию взаимодействия экранирующей оболочки иона с полем атомов в ближайших узлах решетки. Кроме этого, получено условие вырождения квантового состояния электрона в поле экранированного иона, которое существенно упрощает определение энергии основного состояния электрона.

Основные положения работы были доложены 13.02.2020 г. на семинаре теоретического отдела им. Л.М. Бибермана в Институте высоких температур РАН и получили положительные отзывы.

\section{2. Поле экранированного иона в модели Брандта-Китагавы}

Модель экранированного иона изначально была предложена в [1] для решения задачи торможения при движении атомов ионов в электронном га- 
зе. В этой модели распределение $N$ электронов экранирующей оболочки иона с зарядом $Z$ определяется соотношением

$$
\rho_{e i}=\frac{N}{4 \pi \lambda_{N}^{3}} \frac{\lambda_{N}}{r} \exp \left(-\frac{r}{\lambda_{N}}\right), \quad \lambda_{N}=0.48 \frac{(N / Z)^{2 / 3}}{1-N / 7 Z} \cdot \frac{a_{0}}{(Z)^{1 / 3}},
$$

где $\rho_{e i}$ - нормированное распределение электронной плотности, $\lambda_{N}-$ параметр экранировки, $a_{0}-$ боровский радиус. Для однозарядного иона углерода в решетке графена $Z=6, N=1, \lambda_{N} \approx 0.2655 a_{0}$. Ниже используется переобозначение $\lambda_{N} \rightarrow \lambda_{0}$. Из (1) следует, что распределение поля однозарядного иона имеет вид

$$
U_{i}(r)=\frac{Z}{r}-(Z-1)\left[\frac{1}{r} \int_{0}^{r} \rho(r) r^{2} d r+\int_{r}^{\infty} \rho(r) r d r\right]=\frac{1}{r}+\frac{Z-1}{r} \exp \left(-\frac{r}{\lambda}\right) .
$$

Как показали численные эксперименты [3,4], непосредственное использование формулы (2) с параметром экранирования, взятым для однозарядного иона углерода из работы [1], при вычислении энергии основного состояния методом возмущения и вариационным методом приводили к сильному разбросу значений энергий. Как показал дальнейший анализ, это связано с неоднородностью поля. Потенциал поля иона (2) не удовлетворяет теореме вириала для кулоновского поля

$$
\frac{\partial}{\partial r} U_{i}(r) \neq-\frac{U_{i}(r)}{r}
$$

В этом случае, согласно теореме вириала в неоднородном поле, полная энергия финитного движения электрона будет определяться соотношением [6]

$$
\hat{E}(r)=\frac{r}{2} \frac{\partial}{\partial r} U_{i}(r)+U_{i}(r) .
$$

Таким образом, оператор полной энергии для потенциала (2) примет вид

$$
\hat{E}(r)=\frac{U_{i}(r)}{2}+\frac{Z-1}{2 \lambda} \exp \left(-\frac{r}{\lambda}\right) .
$$

На рис.1 приведены распределения потенциала иона и энергии, соответствующие различным значениям параметра экранирования. Из рис.1 видно, что неоднородность поля создает внутренний потенциальный барьер, который увеличивает энергию связи электрона в $s$ состоянии и уменьшает энергию связи этого электрона в $p$ состоянии. Таким образом, неоднородность поля играет затвора, регулирующего локализацию и делокализацию слабосвязанного электрона, не участвующего в образовании валентной связи. 


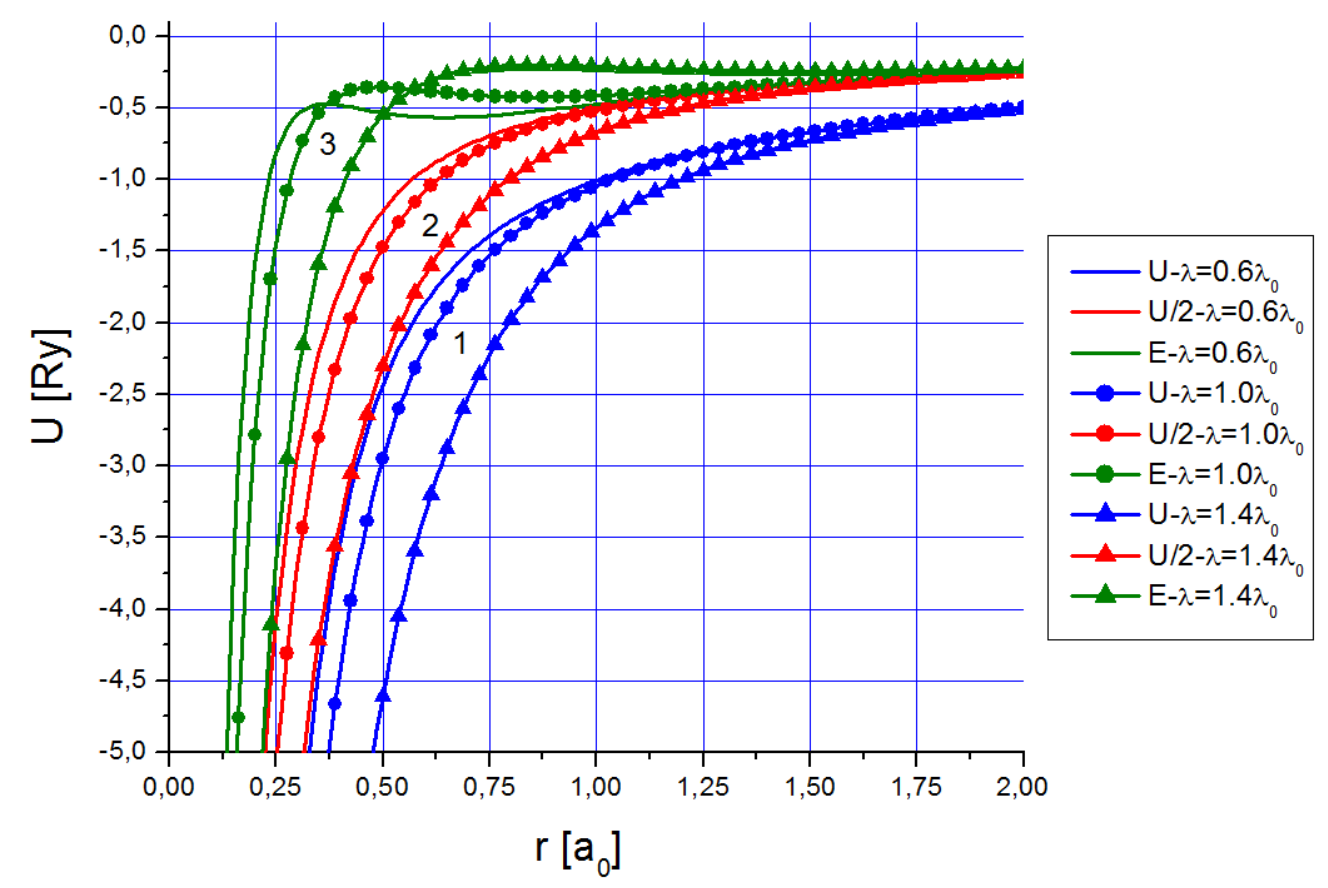

Рис.1. Распределения потенциала иона $(-U)$ (цифра 1) и операторов полной энергии в однородном (-U/2) (цифра 2) и неоднородном $(-E)$ (цифра 3$)$ случаях при различных значениях параметра экранирования.

\section{3. Условие вырождения квантового состояния водородоподобного ато-} ма в неоднородном поле экранированного иона

Получим теперь условие вырождения квантового состояния водородоподобного атома в поле экранированного иона.

Так как для основного состояния атома углерода радиальное уравнение Шредингера имеет два линейно независимых решения $R_{2 s}(r)$ и $R_{2 p}(r)$, то условием вырождения водородоподобного атома в экранированном поле иона (2) будет равенство полных энергии для этих волновых функций

$$
<R_{2 s}^{*}(r) \hat{E}_{i}(r) R_{2 s}(r)>=<R_{2 p}^{*}(r) \hat{E}_{i}(r) R_{2 p}(r)>.
$$

После подстановки выражения (5) и перехода к безразмерной переменной интегрирования это условие примет вид уравнения относительно безразмерной переменной $\chi=1+(n /(2 q \lambda))$, где $q$ - масштабный множитель волновых функций и $n$ главное квантовое число ( $n=2$ для углерода):

$$
\begin{aligned}
& \int_{0}^{\infty}\left[\frac{1}{2} R_{2 s}^{2}(\xi)-\frac{1}{24} R_{2 s}^{2}(\xi)\right]\left[\frac{1}{\xi}+\frac{Z-1}{\xi} \exp (-\chi \xi)\left(1-\frac{\xi}{\bar{\lambda}}\right)\right] \xi^{2} d \xi=0, \\
& \xi=\frac{2 q}{n} r, \quad \bar{\lambda}=\frac{2 q}{n} \lambda, \quad \chi=1+\frac{1}{\bar{\lambda}}, \quad n=2 .
\end{aligned}
$$

После выполнения интегрирования это уравнение примет вид 


$$
\chi^{3}-6 \chi^{2}+9 \chi-4=0
$$

Уравнение (8) имеет два решения $\chi=1$ и $\chi=4$, но при $0<\lambda<\infty$ физический смысл имеет только второе. Таким образом, условием вырождения водородоподобного атома углерода в поле экранированного иона будет соотношение, связывающее масштабный множитель волновой функции и параметр экранирования иона

$$
1 /(q \lambda)=3 \text {. }
$$

Так как полученное условие вырождения водородоподобного атома не зависит ни от заряда ядра $Z$, ни от числа электронов в экранирующей оболочке $N$, то оно применимо для всех водородоподобных атомов и ионов легких атомов с одной заполненной оболочкой. Для изолированного атома (или иона) в вакууме $(q=1)$ это соотношение позволяет определить параметр экранировки иона $\lambda$ из равенства полной энергии электрона в поле экранированного иона первому (или соответствующему заряду иону) потенциалу ионизации атома. В рассматриваемом случае атома углерода в решетке графена это соотношение позволяет построить итерационное решение самосогласованной задачи о квантовом состоянии водородоподобного атома в поле экранированного иона с учетом поля аналогичных атомов решетки и внешних источников поля.

В модели Брандта-Китагавы параметр экранирования иона $\lambda$ определяется по средней энергии электронов оболочки иона в электронном газе в теории Томаса-Ферми. Однако известно [7], что теория Томаса-Ферми не достаточно хорошо определяет распределение электронов в оболочке легких атомов. Кроме этого, и поле окружения иона в решетке существенно отличается от поля электронного ферми-газа. Поэтому при использовании значений параметра экранирования иона из [1] требуется ввести поправку на энергию взаимодействии электронов оболочки с полем атомов в ближайших узлах решетки. По нашим оценкам с учетом этой поправки погрешность расчета энергии основного состояния электрона поле экранированного иона (2) методом возмущения и вариационным методом не превысит 1\%.

\section{4. Определение параметра экранирования иона в решетке графена}

Другой подход к определению параметра экранирования $\lambda$ связан с решением вариационной задачи на экстремум энергии взаимодействия экранирующей оболочки иона с полем ближайших атомов решетки $-U_{s}(r)$, относительно $\lambda$ при сохранении условия вырождения (7). Для этого, учитывая структуру решения уравнения Лапласа в сферической системе коорди- 
нат, представим поле $U_{S}(r)$ в виде первого члена разложения потенциала $U_{s}(r)$ на сфере радиусом равным половине шага решетки $r_{s}=s / 2$ :

$$
V_{s}(r)=\bar{U}_{s}\left(r_{s}\right)\left\{\begin{array}{cc}
\frac{r}{r_{s}}, & r \leq r_{s} ; \\
\pm \frac{r_{s}}{r}, & r \geq r_{s} .
\end{array}\right.
$$

Знак "+" соответствует симметричному $(v+)$, знак "-" - антисимметричному $(v-)$ продолжению поля вне сферы. С учетом этого разложения, энергия взаимодействия оболочки иона с полем окружения $E_{i s}$ будет определяться соотношением

$$
\begin{aligned}
& E_{i s}(\lambda)=-\int_{0}^{\lambda} \rho(r) V_{s}(r) r^{2} d r+\int_{\lambda}^{\infty} \rho(r) V_{s}(r) r^{2} d r \\
& \rho(r)=\frac{Z-1}{\lambda^{2} r} \exp \left(-\frac{r}{\lambda}\right), \\
& \bar{U}_{S}\left(r_{s}\right)=\frac{1}{4 \pi} \oiint_{\Omega} \sum_{v=1}\left\{\bar{U}_{i}\left[R_{v}\left(r_{s}, \theta, \varphi\right)\right]-\bar{U}_{e}\left[R_{v}\left(r_{s}, \theta, \varphi\right)\right]\right\} d \Omega, \\
& \bar{U}_{i}(r)=\frac{1}{r}+\frac{Z-1}{r} \exp \left(-\frac{r}{\lambda}\right), \\
& \bar{U}_{e}(r)=\frac{1}{r} \int_{0}^{\bar{r}} R_{2 s}^{2}(\xi) \xi^{2} d \xi+\frac{\bar{r}}{r} \int_{0}^{\bar{r}} R_{2 s}^{2}(\xi) \xi d \xi, \quad \bar{r}=\frac{2 q}{n} r .
\end{aligned}
$$

Проанализируем теперь зависимости $E_{i s}(\lambda)$ на рис.2. При малых $\lambda$ оба варианта дают близкое положение минимума $E_{i s}$ и среднего потенциала окружения $V_{s}$. Но с увеличением $\lambda$ поведение $E_{i s} v+$ и $E_{i s} v-$ принципиально различно. В варианте симметричного продолжения $E_{i s} v+$ убывает и достигает второго минимума, в то время как в варианте антисимметричного продолжения $E_{i s} v$ - возрастает и, проходя через 0 , достигает максимума примерно при одинаковых значениях $\lambda$, что и минимум $E_{i s} v+$. Следует отметить, что экстремальные значения $E_{i s} v+$ и $E_{i s} v$ - находятся вблизи второго минимума $V_{s}$ при $\lambda>s / 2$. Если прохождение $E_{i s} v$ - через 0 можно трактовать как условие делокализации электрона, то вариант антисимметричного продолжения можно считать физически оправданным приближением, т.к. он приводит к делокализации электрона при радиусе экранирования $\lambda$ меньше половины шага решетки, что можно ожидать для ковалентной связи. А вариант 
с симметричным продолжением можно отнести к молекулам с ионной связью. Таким образом, из приведенного на рис.2 численного решения вариационной задачи следует, что вырожденное состояние слабосвязанного электрона определяется параметром экранирования $\lambda_{m}=0.2 a_{0}$. Это позволяет, с учетом условия вырождения (9), рассчитать самосогласованную энергию основного состояния слабосвязанного электрона в решетке графена:

$$
\begin{aligned}
& E_{m}=\frac{1}{2} \int_{0}^{\infty} R_{2 s}^{2}(\xi)\left[\frac{1}{\xi}+\frac{Z-1}{\xi} \exp (-\chi \xi)\left(1-\frac{\xi}{\bar{\lambda}_{m}}\right)\right] \xi^{2} d \xi, \\
& q_{m}=\frac{1}{3 \lambda_{m}}, \quad \xi=\frac{2 q_{m}}{n} r, \quad \bar{\lambda}=\frac{2 q_{m}}{n} \lambda_{m}, \quad n=2 .
\end{aligned}
$$

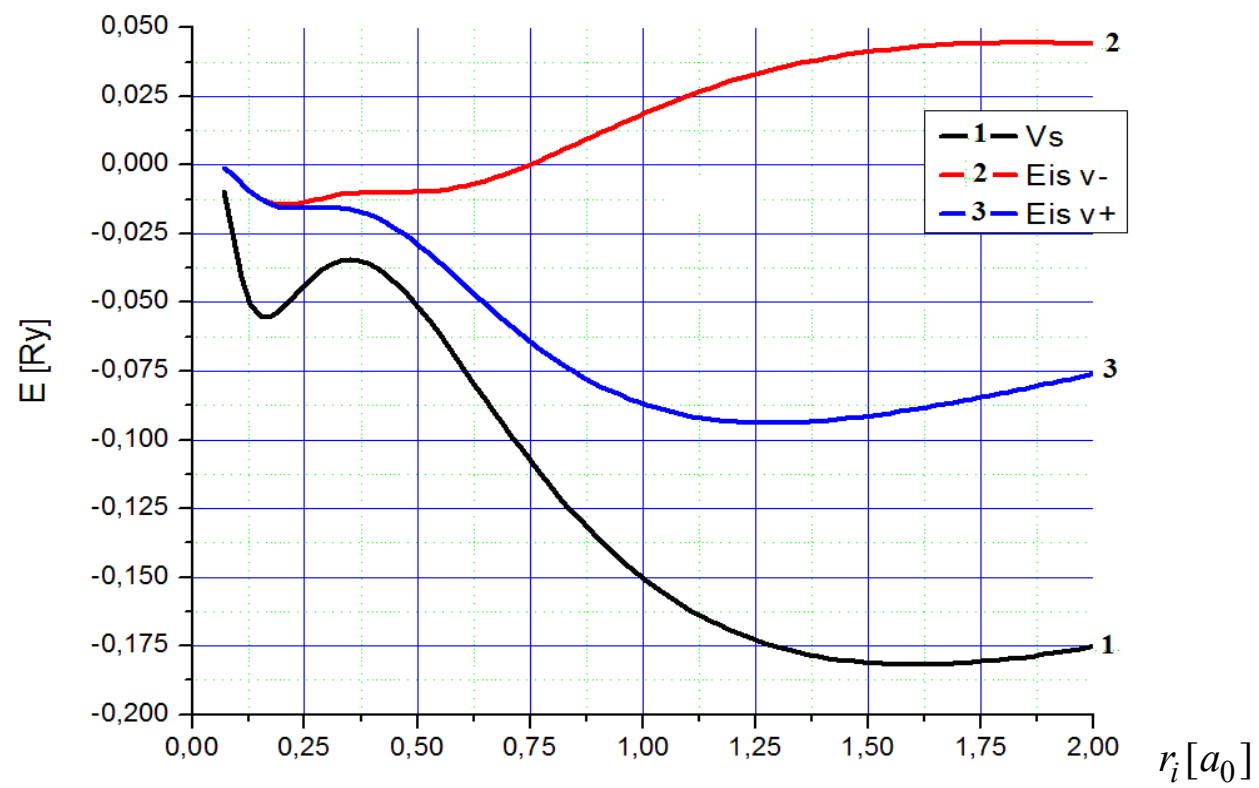

Рис.2. Зависимость среднего потенциала окружения $V_{s}$ и энергий взаимодействия оболочки иона с полем окружения в симметричном $E_{i s} v+$ и антисимметричном $E_{i s} v$ - случае от параметра экранирования $\lambda$.

\section{5. Снятие вырождения слабосвязанного электрона водородоподобного атом в поле атомов в ближайших узлах решетки}

Полученное выше значение параметра экранирования $\lambda_{m}$ и эффективного заряда иона $q_{m}$ основного состояния слабосвязанного электрона позволяют рассчитать энергию взаимодействия этого электрона с полем аналогичных атомов в ближайших узлах решетки. Для этого в аппроксимации потенциала поля окружения на сфере $r_{s}=s / 2$ необходимо учесть угловые члены разложения. В этом случае при антисимметричном приближении оператор возмущении поля экранированного иона примет вид [8]: 


$$
\begin{aligned}
& \hat{U}_{s}(r, \theta, \varphi)= \begin{cases}V_{s} \frac{r}{r_{s}}+\sum_{l, m} C_{l, m}\left(r / r_{s}\right)^{l} Y_{l m}(\theta, \varphi), & r \leq r_{s} ; \\
-V_{s} \frac{r_{s}}{r}-\sum_{l, m} C_{l, m}\left(r_{s} / r\right)^{l} Y_{l m}(\theta, \varphi), & r \geq r_{s} ;\end{cases} \\
& C_{l, m}=\oiint_{\Omega} \sum_{v=1}^{N} U_{s v}\left(r_{s}, \theta, \varphi\right) Y_{l m}(\theta, \varphi) d \Omega .
\end{aligned}
$$

В табл.1 представлены коэффициенты разложения поля трех, 9-ти и 12-ти ближайших атомов на сфере радиуса $r_{s}=s / 2$. Из таблицы видно, что в отсутствии внешнего поля изменения параметров разложения волновой функции электрона связаны со средним значением потенциала на этой сфере $V_{s}$ и угловым членом разложения, пропорциональным $Y_{20}(\theta)$. Первый определяет только энергию состояния, а второй приводит к снятию орбитального вырождения. Таким образом, оператор возмущения принимает вид

$$
\hat{U}_{s}(r, \theta) \approx V_{s}(r)+0.5 \cdot \tilde{U}_{s 2}(r)\left(3 \cos ^{2} \theta-1\right) .
$$

Таблица 1. Коэффициенты разложения поля атомов окружения при учете $k_{m}$ ближайших узлов решетки графена.

\begin{tabular}{|l|c|c|c|c|c|}
\hline $\begin{array}{l}\text { Сферическая } \\
\text { функция }\end{array}$ & $Y_{00}$ & $Y_{10}$ & $Y_{11}$ & $Y_{1-1}$ & $Y_{20}$ \\
\hline $\begin{array}{l}\text { Коэффициент } \\
\text { разложения } k_{m}=3\end{array}$ & -0.01942 & $-4.811 \mathrm{E}-18$ & $7.177 \mathrm{E}-005$ & $1.661 \mathrm{E}-008$ & 0.19680 \\
\hline $\begin{array}{l}\text { Коэффициент } \\
\text { разложения } k_{m}=9\end{array}$ & -0.03848 & $-4.811 \mathrm{E}-18$ & $7.177 \mathrm{E}-005$ & $1.661 \mathrm{E}-008$ & 0.18588 \\
\hline $\begin{array}{l}\text { Коэффициент } \\
\text { разложения } k_{m}=12\end{array}$ & -0.04411 & $1.068 \mathrm{E}-016$ & $7.171 \mathrm{E}-005$ & $1.601 \mathrm{E}-008$ & 0.153664 \\
\hline
\end{tabular}

Так как для атома на плоскости есть выделенное направление, то волновые функции следует брать в параболической системе координат. Однако при вычислении энергии взаимодействия удобно использовать их представление в сферической системе. Вычислим изменение энергии для двух волновых функций в параболической системе координат

$$
\psi_{100}(\xi, \eta)=\frac{1}{\sqrt{2}}\left[R_{s}(r)+R_{p}(r) Y_{10}(\theta)\right], \quad \psi_{001}(\xi, \eta)=R_{p}(r) Y_{11}(\theta, \phi) .
$$

Для этих волновых функций энергия невырожденного состояния с учетом поля окружения будет определяться соотношениями 


$$
\begin{aligned}
& E_{100}=E_{0}+E_{1 s p}-E_{2 s}, \quad E_{001}=E_{0}+E_{1 p}+E_{2 s}, \\
& E_{0}=<\psi^{*}\left[\frac{r}{2} \frac{\partial}{\partial r} U_{i}\left(r, \lambda_{1}\right)+U_{i}\left(r, \lambda_{1}\right)\right] \psi>, \\
& E_{1 s}=\frac{1}{2} \int_{0}^{\infty}\left[R_{2 s}^{2}(\xi)+R_{2 p}^{2}(\xi)\right] \tilde{U}_{s 1}\left(r, \lambda_{1}\right) \xi^{2} d \xi=\int_{0}^{\infty} R_{2 p}^{2}(\xi) \tilde{U}_{s 1}\left(r, \lambda_{1}\right) \xi^{2} d \xi, \\
& E_{2 s}=\int_{0}^{\infty} R_{2 p}^{2}(\xi) \tilde{U}_{s 2}\left(r, \lambda_{1}\right) \xi^{2} d \xi .
\end{aligned}
$$

В результате вычисления этих интегралов получим невырожденные значения энергии электрона в этих состояниях $E_{10}=-5.55$ эВ и $E_{001}=-5.3619$ эВ. Эти значения энергий находятся в удовлетворительном согласии с экспериментальными значениям работы выхода электронов при фотоэмиссии 4.65.3 эВ с эмиттеров на основе углеродных нанотрубок [9].

\section{6. Заключение}

В работе предложен алгоритм самосогласованного расчета квантового состояния водородоподобного атома с экранированным ионом в поле ближайших атомов решетки. В этой модели водородоподобный атом зависит от двух параметров - эффективного заряда электрона $q$ и параметра (эффективного радиуса) экранирования $\lambda$. В используемой нами модели экранирования Брандта-Китагавы параметр $\lambda$ определяется по средней энергии кулоновского взаимодействия всех электронов $N$ экранирования с зарядом ядpa $Z$ в теории Томаса-Ферми. В нашем алгоритме он определяется по минимуму энергии взаимодействия экранирующей оболочки с полем аналогичных водородоподобных атомов окружения в вырожденном состоянии.

Также в работе было получено условие вырождения квантового состояния водородоподобного атома в неоднородном поле экранированного иона. Это условие определяет соотношение между $q$ и $\lambda$ при равенстве энергий электрона в $s$ и $p$ состояниях в поле экранированного иона. Для расчета энергии взаимодействии экранирующей оболочки и слабосвязанного электрона с полем окружения используется аппроксимация этого поля через его угловое разложение на сфере радиусом $r_{s}$, равным половине шага решетки $s / 2$. Как показали расчеты, в отсутствии внешних источников для плоской решетки существенное значение имеют только два члена этого разложения - среднее значение потенциала на сфере и член, пропорциональный $Y_{20}(\theta)$. Таким образом, при расчете энергии взаимодействии экранирующей оболочки $E_{\text {is }}$ учитывается только среднее поле.

Как показали расчеты, зависимости энергии взаимодействия экрани- 
рующей оболочки $E_{i s}$ от радиуса экранирования $\lambda$ определяются симметрией продолжения аппроксимации поля окружения через сферу разложения $r_{s}$. Причем только при антисимметричном продолжении величина $E_{i s}$ проходит через ноль, что можно трактовать как переход от растяжения экранирующей оболочки внешним полем к её сжатию.

При расчете снятия вырождения квантового состояния слабосвязанного электрона при его взаимодействии с полем окружения учитывалось и среднее поле на сфере $r_{s}$ и его угловое распределение. Для волновых функций в параболической системе координат угловое распределение приводит расщеплению вырожденного состояния на два состоянии с дипольным и квадрупольным моментом. Значения энергий этих состояний находятся в удовлетворительном согласии с работой выхода при фотоэмиссии эмиттеров на основе нанотрубок. Разработанные алгоритмы и программы будут использованы для расчета режимов работы и спектра эмиссии таких эмиттеров.

\section{СПИСОК ЛИТЕРАТУРЫ}

1. W. Brandt, M. Kitagawa. Effective stopping-power charges of swift ions in condensed matter // Phys. Rev., 1982, v.B25, № 9, p.5631-5637.

2. Б.Г. Фрейнкман. Модель псевдопотенциала атома углерода в решетке графена // Математическое моделирование, 2015, т.27, № 7, с.122-128;

B.G. Freinkman. Model psevdopotentsiala atoma ugleroda v reshetke grafena // Matematicheskoe modelirovanie, 2015, t. 27, № 7, s.122-128.

3. B. Freinkman, S. Polyakov, I. Tolstov. A variational solution of the Schrödinger equations in an inhomogeneous Coulomb field proceeding international scientific conference (Matmodel 17) 13.12-16.12, 2017. - Borovets: Bulgaria.

4. I. Tolstov, B. Freinkman, S. Polyakov. Variational Solution of the Schredinger Equation in an Inhomogeneous Central Field // EPJ Web of Conferences, 2018, v.173. Art. 03022 (4p.). DOI: $10.1051 /$ epjconf $/ 201817303022$

5. B. Freinkman, S. Polyakov, I. Tolstov. The variational solution of the Schredinger equation for a hydrogen-like carbon atom in a graphenelattice / Math. Modeling and Comp. Physics, 2019: Abstracts of Int. Conf. (High Tatra Mountains, Slovakia, 01.07-05.07, 2019).

6. В.А. Фок. Начала квантовой механики. - M.: URSS, 2013. 376 с.; V.A. Fok. Nachala kvantovoi mekhaniki. - M.: URSS, 2013, 376 s.

7. П. Гамбош. Статистическая теория атома и ее применение. - М.: ИЛ, 1951, 398 c.; P. Gambosh. Statisticheskaya teoriya atoma i ee primenenie. - M.: IL, 1951, $398 \mathrm{~s}$.

8. J.D. Jackson. Classical Electrodynamics, third ed. - NY: John Wiley\&Sons, Inc., 1999, 832p.

9. А.В. Елеикий. Холодные полевые эмиттеры на основе углеродных нанотрубок // УФН, 2010, т.180, вып 9, с.897;

A.V. Eletskii. Carbon nanotube-based electron field emitters // Physics-Uspekhi, 2010, v. 53, p. $863-892$. 